\title{
Contactless electromagnetic measuring system using conventional calibration algorithms to determine scattering parameters
}

\author{
T. Zelder, H. Rabe, and H. Eul
}

Institut für Hochfrequenztechnik und Funksysteme, Universität Hannover, Appelstraße 9A, 30167 Hannover, Germany

\begin{abstract}
In this paper, a contactless measuring system for the determination of the S-parameters of planar circuits is presented. With a contactless measuring system it is possible to characterise a device-under-test (DUT) embedded in a planar circuit environment without cutting the planar transmission lines connecting the DUT. The technique utilizes four identical capacitive probes in conjunction with a vector network analyser (VNA). For the usage of electromagnetic probes compared to other coupling techniques like the electro-optic probing, there is no need for expensive and complex equipment in addition to the typical equipment of a common microwave laboratory. The S-parameters are determined accurately using conventional calibration methods. A simple analytical model for the representation of the basic characteristics is developed. Furthermore, the influences on the S-parameters as a result of a variation in the coupling are presented. With the knowledge of the system characteristics, an accurate contactless measurement system is set up. The comparison between conventional and contactless measurements in a frequency range of $1-20 \mathrm{GHz}$ shows a very good agreement with a phase error smaller than $1^{\circ}$.
\end{abstract}

\section{Introduction}

For the development of complex microwave planar systems, consisting of multiple circuits, it is necessary to measure the S-parameters of each circuit separately. Thus, the system performance for the different circuits can be analysed and verified individually. However, with a conventional measurement setup and the use of coplanar probes such a separate characterisation is only possible with physical modifications of the planar system, like cutting the planar transmission lines connecting the device-under-test (DUT). Only

Correspondence to: T. Zelder

(zelder@hft.uni-hannover.de) by contactless measurement techniques the characterisation of every single circuit of the entire planar system can be realised. Further on, a contactless system has the advantage that it works wearless. The probe dimension is small with respect to conventional coplanar probes. Also, fast testing becomes possible, due to the not required contacting of the DUT with the probing system.

Some different contactless measurement methods for the characterisation of two-ports embedded in planar circuits already exist in the literature. The different contactless techniques have in common that the test signals are supplied to the planar system by conventional coplanar probes or coaxial interfaces. For the determination of the S-parameters, it is necessary to measure the forward and backward travelling electromagnetic waves at the interface planes of the DUT. The measurement of these complex waves is performed contactlessly.

The various contactless techniques are based on different physical principles as e.g. electro-optic probing (David et al., 1995; Mertin et al., 1992), electron-beam probing (Zlobin, 1993), photoemissive probing (Seitz et al., 1990), electric force microscopy (Tabib-Azar and Wang, 2004; Bridges, 2004), electromagnetic probing (Gao and Wolff, 1998). An overview of the different physical principles is given in Mertin et al. (1998) and Sayil et al. (2005).

In this work, a contactless electromagnetic probing system with four capacitive probes is used. Compared to the other contactless coupling techniques, it has the advantage that no expensive and complex equipment is needed.

In addition, four different calculation methods exist for the contactless determination of the S-parameters: the method of comparison, the method of function minimisation, the method of contactless sampled-line reflectometry and the contactless vector network analysis.

The method of comparison requires a single planar transmission line terminated by its characteristic impedance. The propagating wave along the planar transmission line is mea-

Published by Copernicus Publications on behalf of the URSI Landesausschuss in der Bundesrepublik Deutschland e.V. 


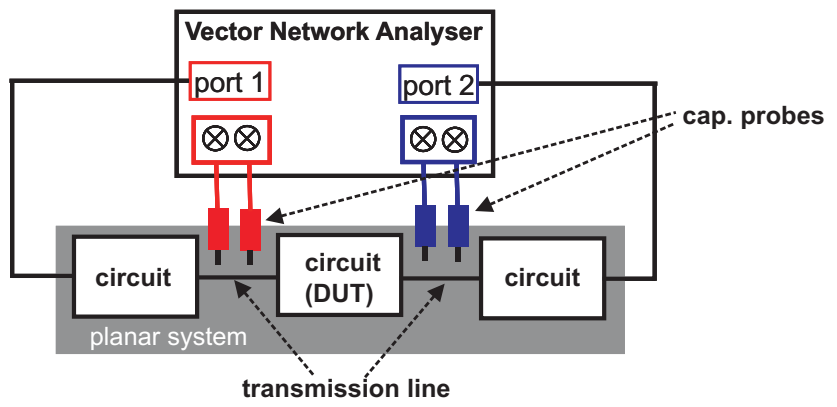

Fig. 1. Principle contactless measuring system using a two-port VNA.

sured by coupling a part of the wave to a sensing receiver. Subsequently, the DUT is inserted into the transmission line, and a part of the energy in front of and behind the DUT is coupled contactlessly to the receiver. After that, the S-parameters are computed according to Dudley et al. (1999).

For the method of function minimisation, the complex amplitudes of the waves propagating along the transmission lines in front of and behind the DUT are measured contactlessly at different discrete positions (Osofsky and Schwarz, 1992). Subsequently, the forward and backward travelling waves are calculated using a function minimisation method e.g. the least square (Gao and Wolff, 1997) or the simplex method (Quardirhi and Laurin, 2003).

In Hui and Weikle (2005) a contactless sampled-line (sixport) reflectometer is used to determine the reflection coefficient of the DUT in the frequency range from $700 \mathrm{MHz}$ to $1.2 \mathrm{GHz}$. The measurement setup consists of a signal source, one planar transmission line, a one-port DUT and four contactless power detectors. The power detectors are used to sample the standing waves at four fixed positions along the transmission line. After the six-to-four-port reduction (Engen, 1978) a calibration is carried out (Schiek, 1999).

The fourth method to determine the S-parameters is the contactless vector network analysis. This method uses a VNA, contactless probing equipment and a conventional calibration algorithm. A practical realisation for one-port measurements with the use of the SOL calibration (Schiek, 1999) is shown in Stenarson et al. (2001). There an inductive and a capacitive probe are used. Both probes are positioned along a transmission line, which on the one side is fed by a signal source and on the other side can be terminated by the calibration standards or the DUT. In the frequency range from $700 \mathrm{MHz}$ to $14 \mathrm{GHz}$ a comparison between the contacted and the contactless network analysis showed a good agreement. One advantage of the contactless vector network analysis compared to the contactless sampled-line reflectometer is a twice higher dB-dynamics range, due to the heterodyne reception principle of the VNA.

The measuring system in Stenarson et al. (2001) served as an initial point for the contactless system described here. The contactless system presented in this paper comprises only capacitive coupling structures. Further on, this paper focuses on the derivation of a simple analytical model that accounts for the basic characteristics of the contactless probing system. Additionally the test system is expanded to the characterisation of two-ports. Thereby, a comparison between the conventional and the contactless network analysis is performed. For the contactless network analysis in combination with the electromagnetic probes a high accuracy can be achieved which is shown in this paper.

\section{The contactless measurement system}

The principle setup of the contactless measurement system is illustrated in Fig. 1. A microwave planar network consisting of e.g. three circuits and a two-port VNA with four measuring channels is shown. The planar network is fed by the ports of the VNA. The circuits are connected to planar transmission lines. The S-parameters of the DUT in the centre of the planar system shall be determined. Thereby, four capacitive probes are used. Two probes are located in front of the DUT and two behind of it.

The internal couplers of the VNA are replaced by the contactless coupling structures. Thus, the rough separation of the forward and backward travelling waves at the measuring channels is not existent anymore. The coupled energy is now directly connected to the four measuring channels of the VNA. The voltages coupled by the contactless probes are linear combinations of the forward and backward travelling waves in front of and behind the DUT. By means of conventional calibration methods the S-parameters of the DUT can be determined. Thereby, it is necessary to use the same planar transmission line geometry during calibration and measurement, so that the influences of the contactless probes on the transmission line are identical for both cases.

To analyse the characteristics of the measuring system, a simple one-port model is used. The description of the model and the simulation results are given in Sects. 3 and 4 of this paper.

\section{A basic model of the contactless system}

For the analysis of the system characteristics, a simple analytical model is developed. It is shown in Fig. 2.

The capacitive coupling structure is modelled by two identical capacitances $C_{1}$. A lossless $50 \Omega$ transmission line of length $l_{1}$ and permittivity $\epsilon_{r}=1$ is located between the two capacitances. The transmission line is fed by a test signal with a power level of $1 \mathrm{dBm}$. The termination is located at the end of another lossless $50 \Omega$ transmission line. This termination may be a calibration standard or the DUT. The capacitive coupled voltages are determined in a $50 \Omega$ system at the measuring channels $m_{1}$ and $m_{2}$ (see Fig. 2). Additive 


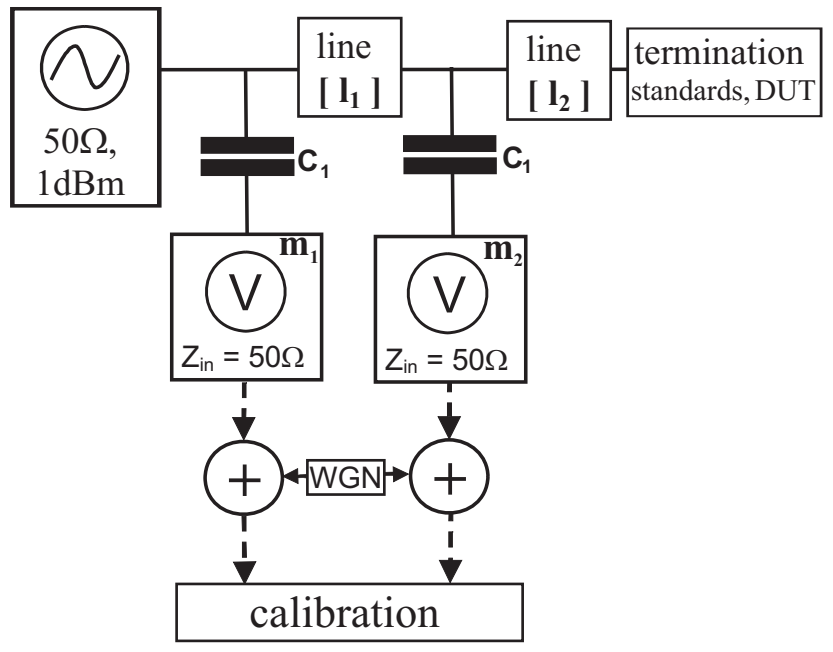

Fig. 2. Simple simulation model used for basic analysis of the contactless system.

white Gaussian noise (AWGN) was applied at the receiver with a respective power level of $-118 \mathrm{dBm}$. Thereby, the ratio $m_{1} / m_{2}$ with the added noise is averaged over 500 values. The error terms of the system are determined applying the SOL calibration with a $50 \Omega$ load, a short and an open standard.

\section{System characteristics}

In this section the essential system characteristics are surveyed. At first the optimal probe distance is considered. After that, it is shown that the contactless system can be used to characterise a DUT embedded in a planar circuit environment. At least, the effects on the measurement results after changing the coupling coefficient are analysed.

\subsection{The optimal probe distance}

For the dimensioning of an effective measurement system the optimal distance between the two probes is important to know. For this analysis the probe distance $l_{1}$ is varied for a constant frequency of $f=1.5 \mathrm{GHz}$. After the calibration the $50 \Omega$ calibration standard is used as a DUT. The results are presented in Fig. 3.

In Fig. 3 the absolute values of the S-parameter $S_{11}$ in $\mathrm{dB}$ are illustrated as a function of the ratio of the probe distance $l_{1}$ to the wavelength. The maximum dynamic is reached at odd multiples of the probe distance $\lambda / 4$. At integer multiples of $\lambda / 2$, the dynamic range clearly decreases. There, the ratio of the complex voltages of both probes is nearly the same for different calibration standards.

In Fig. 4, the reflection coefficient of the $50 \Omega$ calibration standard is plotted against the frequency.

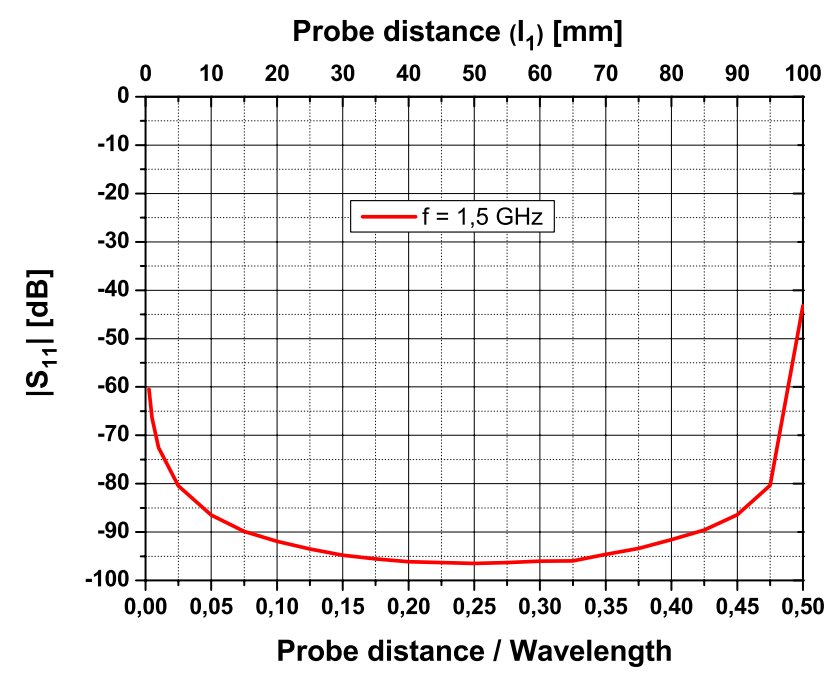

Fig. 3. S-parameters as a function of the distance $l_{1}$, DUT: $50 \Omega$ load.

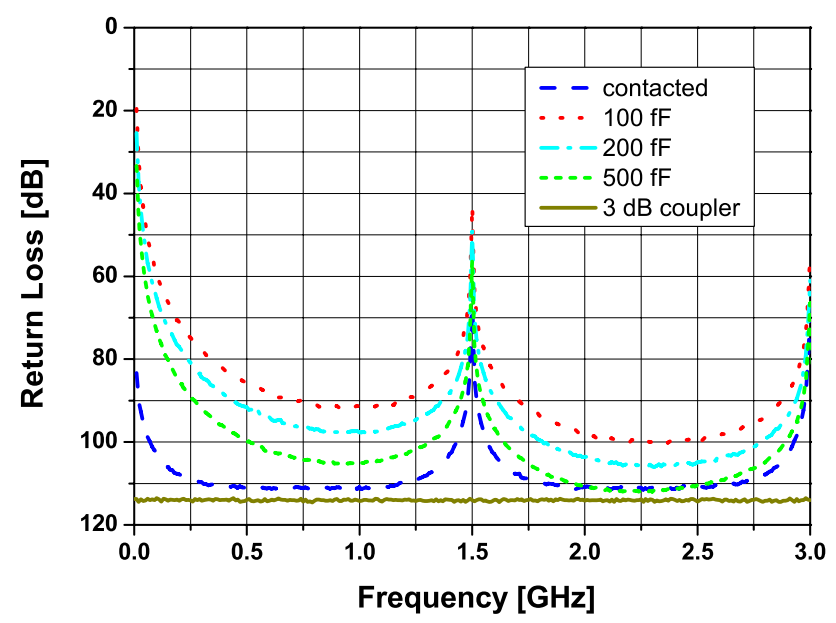

Fig. 4. S-parameters against the frequency after changing the distance between the probes and microstrip line, DUT: $50 \Omega$ load.

A probe distance of $l_{2}=100 \mathrm{~mm}$ is used. In theory, the optimal distance between the probes is achieved at odd multiples of $f=750 \mathrm{MHz}$. However, the areas of the highest dynamics ranges in Fig. 4 are located slightly above $750 \mathrm{MHz}$ and $2.25 \mathrm{GHz}$. They are slightly shifted because of the increasing coupling at higher frequencies. Also, different capacitances $C_{1}$ are examined and illustrated by dotted lines. As expected, the system dynamic range decreases with a decreasing coupling factor. For the comparison with the conventional network analysis method, the capacitive coupling structure is replaced by an ideal coupler. The curve of the reflection coefficient with the use of a 3-dB coupler is illustrated in Fig. 4, yielding a constant dynamic range of $114 \mathrm{~dB}$ in the entire frequency interval. 


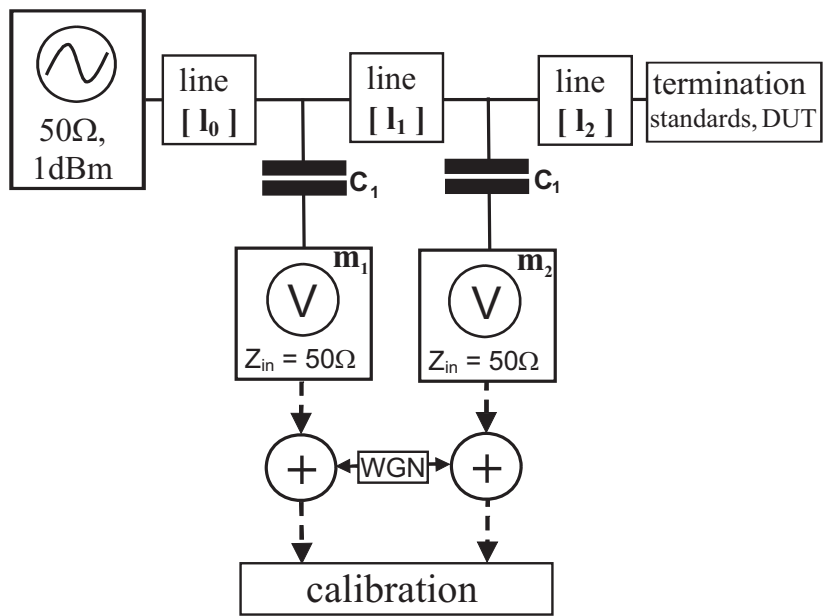

Fig. 5. Enhanced simulation model.

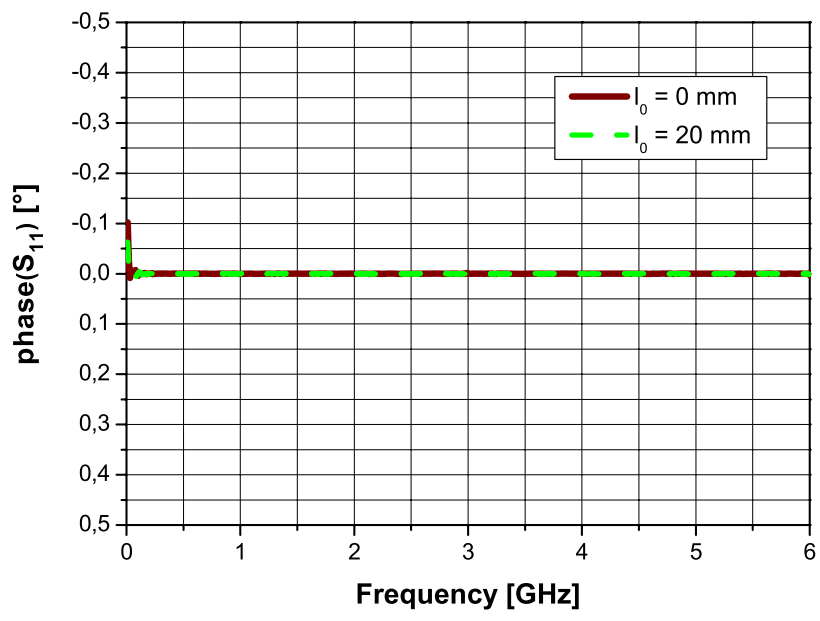

Fig. 6. Phase of $S_{11}$ after the introduction of a transmission line $\left(l_{0}\right)$ between the source and the first probe, DUT: Open.

\subsection{Characterisation of embedded circuits}

Now, it will be tested, if the contactless system can be used to measure the S-parameters of embedded circuits that are an internal part of a more complex planar circuit. Therefore, after the calibration of the contactless system depicted in Fig. 2, a modification of the simulation setup is to be used. The modified configuration is illustrated in Fig. 5.

A new lossless transmission line with the length $l_{0}$ is inserted between the signal source and the first transmission line $\left(l_{1}\right)$ in front of the capacitance $\left(C_{1}\right)$. Subsequently, the calibration standards are measured again with different line lengths $l_{0}$. It results that the calculated S-parameters for different line lengths are the same. For example the phase of $S_{11}$ is illustrated in Fig. 6 for the two line length $l_{0}=0 \mathrm{~mm}$ and $20 \mathrm{~mm}$. In terms of the DUT, the open calibration standard is used. The figure shows, that for different line lengths

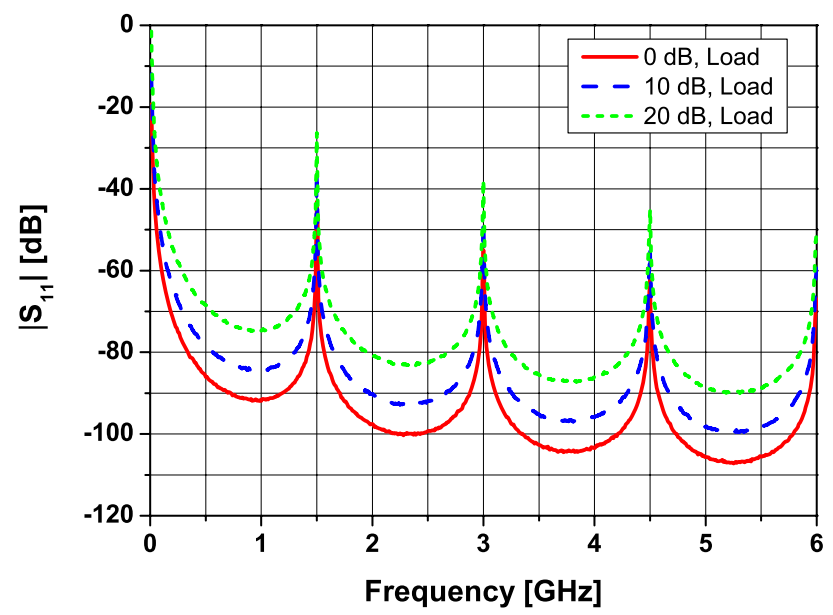

Fig. 7. S-parameters after the introduction of an attenuator between the source and the first probe, DUT: $50 \Omega$ load.

$l_{0}$ the correct phase of the DUT is determined.

After that, instead of transmission line $l_{0}$ an attenuator is used in the modified model. The measurement results of the $50 \Omega$ calibration standard are shown in Fig. 7. It can be seen that the dynamic range with an increase of attenuation decreases. The differences in the dynamics correspond to the different attenuator values.

Furthermore, the measurements of the high reflective calibration standards are considered. In this case, the differences of the simulation results between the models with and without an additional attenuator are very small and reducible on the noise influence. This proves that a measurement of a circuit embedded within a planar system is basically possible. However, the system dynamic range is a function of the signal level. If the signal level is low at the coupling positions along the transmission line, for example because of a preceding filter, a measurement is only partially possible.

\subsection{Variations of the coupling coefficient}

Subsequently, the model of Fig. 2 is used to identify the influences on the simulation results derived from inaccurate probe positioning. Therefore, after the calibration the coupling capacitance, $C_{1}$ is changed by $10 \%$ from $100 \mathrm{fF}$ to $110 \mathrm{fF}$. In terms of the DUT, the open calibration standard is used. Figure 8 shows the parameter $\left|S_{11}\right|$ in $\mathrm{dB}$ against frequency.

The curves for four different values of $l_{2}$ are shown in Fig. 8. The error is visible as a result of the differences of the curves in comparison to the ideal 0dB-curve. The number of ripple maxima over the frequency increases with the line length $l_{2}$. The reason is that during the calibration, if a high reflective standard is used as a termination, a standing wave exists along the transmission line $l_{1}$ and $l_{2}$. In dependence of the frequency, voltage- or current zero points arise at the probe positions. The ripple maxima are correlated with 


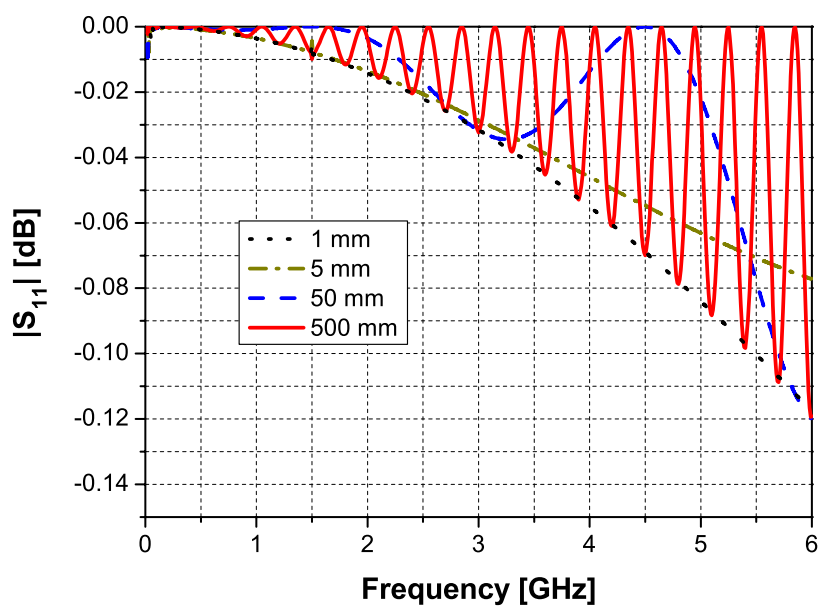

Fig. 8. Variation of the line length $l_{2}$ with changed capacitance $C_{1}$ after the calibration, DUT: Open.

the number of voltage- or current zero points on the transmission line at the reference probe position. Thereby, the reference probe is connected to the receiver $m_{2}$. Furthermore, it is to note that with increasing frequency, the error magnitude increases, as well. The cause of the errors is the changed interaction between the probes and the transmission line due to the different coupling capacitances.

In addition, the distance $l_{2}$ between the probes and the DUT is changed after the calibration. Again the open calibration standard is measured. As expected, the magnitude of $S_{11}$ changes only because of the noise influence. The phase is changing proportional to the length variation $\Delta l$ between the probes according to Eq. (1). Thereby, $f$ stands for the frequency and $v$ is the phase velocity along the line.

$\Delta \varphi=-2 \cdot \frac{2 \cdot \pi \cdot f}{v} \cdot \Delta l$

By means of a simple analytical model the basic system characteristics are analysed. Itis shown, that the $\lambda / 4$ distance is the optimal probe distance. Also, it is pointed out, that the contactless system can be used for the measurement of Sparameters from embedded circuits that are an internal part of a more complex circuit. However, a certain signal level is required for measurements. Further on, if the coupling capacitances change due to environmental effects during the measurement of a high reflective standard, ripples occur in the S-parameters. Thereby, the number of ripples depends of the line length between the probes and the DUT. By means of the known characteristics of this contactless system, an effective contactless measuring system is configured. The measurement setup and results are presented in the following Sects. 5 and 6.

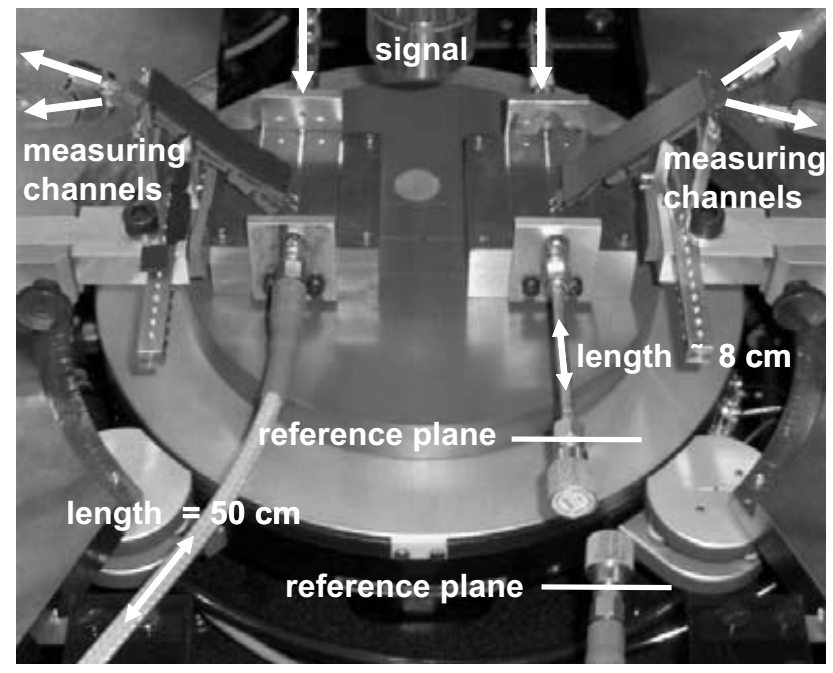

Fig. 9. Photograph of the contactless measurement setup with four capacitive probes in a distance of $4 \mathrm{~mm}$.

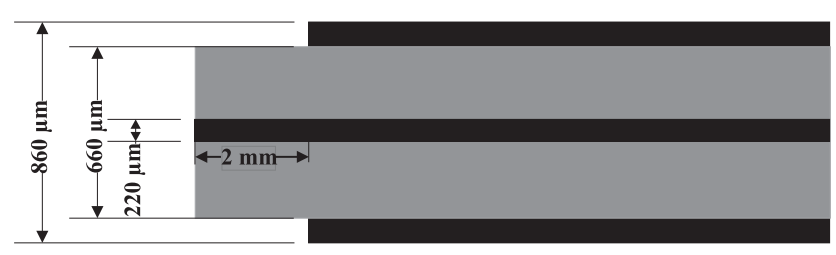

Fig. 10. Cross section of the capacitive probe dimensions.

\section{Measurement setup}

According to the theoretical considerations in the previous sections the measurement system is realised. A picture of the measurement setup is illustrated in Fig. 9.

As VNA, the PNA E8361A of Agilent Technologies was used. The VNA feeds two $50 \Omega$ microstrip lines. For each microstrip line, two capacitive probes are positioned along the planar microstrip lines. The probes are directly connected with flexible coaxial SMA cables to the four measuring channels of the VNA. At the ends of the mircostrip lines two coaxial cables with different lengths $(50 \mathrm{~cm}$ and $8 \mathrm{~cm})$ are used. The two cables are terminated with the calibration standards or the DUT. For a later comparison between the contactless and the contacted measurement method, coaxial reference planes and calibration standards are used.

The probes are realised with semi-rigid coaxial lines by $H U B E R+S U H N E R$ with an inner diameter of $220 \mu \mathrm{m}$. A drawing of a probe is illustrated in Fig. 10. The outer conductor of the semi-rigid line is removed along a length of $2 \mathrm{~mm}$, so that the dielectrics and the inner conductor jut over the coaxial line.

The probe distance is $4 \mathrm{~mm}$, which equals a $\lambda / 4$-distance at around $13.5 \mathrm{GHz}$. Thereby, the effective permittivity of the planar microstrip line is around $\epsilon_{r, e f f} \approx 1.9$. The two ca- 


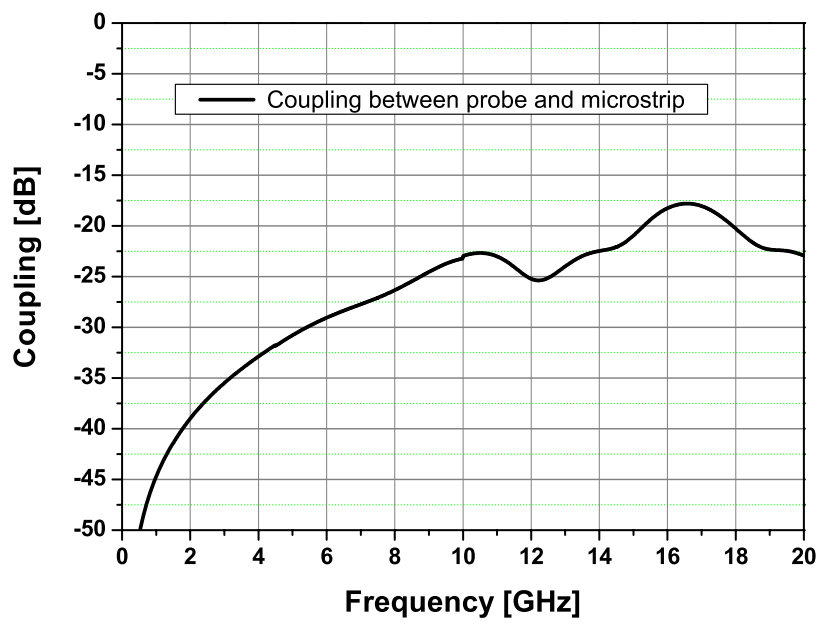

Fig. 11. Coupling between capacitive probe and mircostrip.

pacitive probes are placed in front of and behind the DUT. To get a high coupling coefficient, the probes are positioned with a slant angle of $45^{\circ}$ with respect to the microstrip line. Thereby, the dielectrics of the probes touched the mircostrip line without any electrical contact. By means of Ansoft HFSS an electromagnetic field analysis of one probe above the microstrip line is performed. Figure 11 shows the distribution of the coupling over the frequency. The curve is frequency dependent and shows the typical high-pass performance of the coupling factor. At lower frequencies, the coupling is very small. In a frequency range from 0 to $10 \mathrm{GHz}$ the insertion loss increases up to $22 \mathrm{~dB}$. The effect of the probes on the field distribution of the microstrip is not specified quantitatively, because the influences are already included in the error coefficients of the system, which are calculated by means of the calibration.

For the calibration, SOLT is used. A bandpass filter with coaxial adaptors and a passband between $12-18 \mathrm{GHz}$ is taken as a DUT.

\section{Measurement results}

This chapter is devoted to the comparison of the measurement results between the contactless and the contacted vector network analysis. In Fig. 12, the parameter $\left|S_{11}\right|$ in $\mathrm{dB}$ of the bandpass filter is shown for both systems. A very good agreement of the curves up to $20 \mathrm{GHz}$ is observed. Only in the frequency range below circa $1.1 \mathrm{GHz}$ small variations exist. The reason is the low coupling (see Fig. 11) and the fact that the optimal probe distance of $\lambda / 4$ is far away at these frequencies.

The varieties between the curves of magnitude and phase of the parameter $S_{11}$ is shown in Fig. 13.

The deviations of the magnitudes in a frequency range from $1.1 \mathrm{GHz}$ to $20 \mathrm{GHz}$ are less than $-30 \mathrm{~dB}$. The discrep-

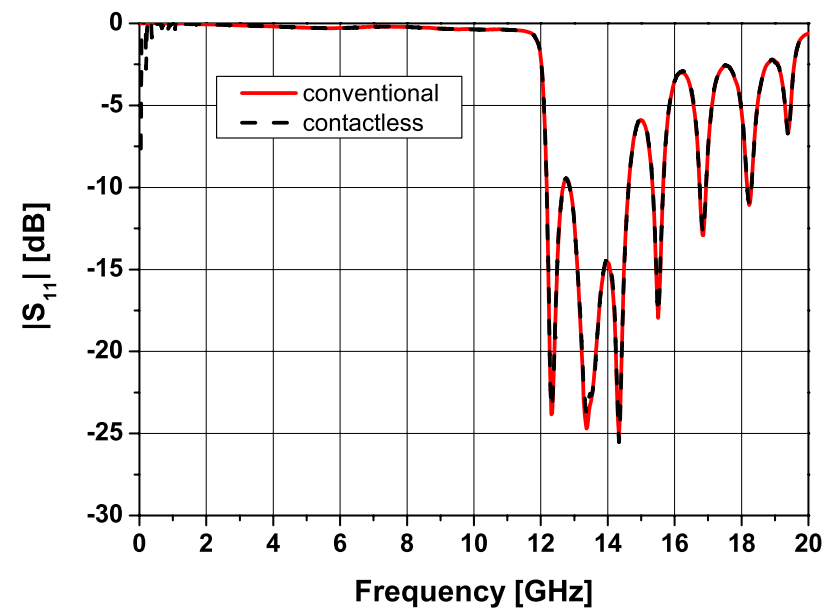

Fig. 12. Comparison of the measurement results between conventional and the contactless measurement method, DUT: Filter.

ancies of the phases in the blocking zone of the bandpass filter between $1.2 \mathrm{GHz}$ and $12 \mathrm{GHz}$ are less than $1^{\circ}$. Different sources of error exist: the radiation of the microstrip lines, errors of the VNA, the repeatability of the calibration and the use of flexible coaxial cables.

The results for the parameter $S_{21}$ of the DUT are shown in Fig. 14 which illustrates the varieties between the contactless and contacted vector network analysis.

A very good agreement between the results of the two measurement methods can be observed. Between $1.25 \mathrm{GHz}$ and $20 \mathrm{GHz}$, the differences are very small yielding magnitude deviations less than $-30 \mathrm{~dB}$. The least deviation appears in the range of the optimal probe distance. The varieties of the phases in the passband are smaller than $1^{\circ}$.

In the next step it is shown, how a modification of the probe position influences on the measured S-parameters. After the calibration the vertical distance between the four probes and the microstrip lines is extended, so that there is no contact between the dielectrics of the probes and the planar transmission lines. Three different vertical distances are examined: $20 \mu \mathrm{m}, 50 \mu \mathrm{m}$ and $100 \mu \mathrm{m}$. The influences on the $\mathrm{S}$-parameters as a result of the changed distances are depicted in the Fig. 15.

Figure 15 shows the difference between the S-parameters $S_{22}$ with and without variation of the distance. For the measurement, the short calibration standard is used. It is obvious that like in the simulation results, ripples occur, which rise with increasing coupling distance. By the shift of $50 \mu \mathrm{m}$, the error is smaller than $\pm 0.1 \mathrm{~dB}$ in a frequency range from $1 \mathrm{GHz}$ to $20 \mathrm{GHz}$. Furthermore, it is noticed that only a small error occurs in the region of the $\lambda / 4$ distance. As it is known from the simulation results, the number of zero points of the standing wave on the transmission line in dependence of the frequency located at the reference probe is correlated with the number of ripples. The zero points arise during the cali- 

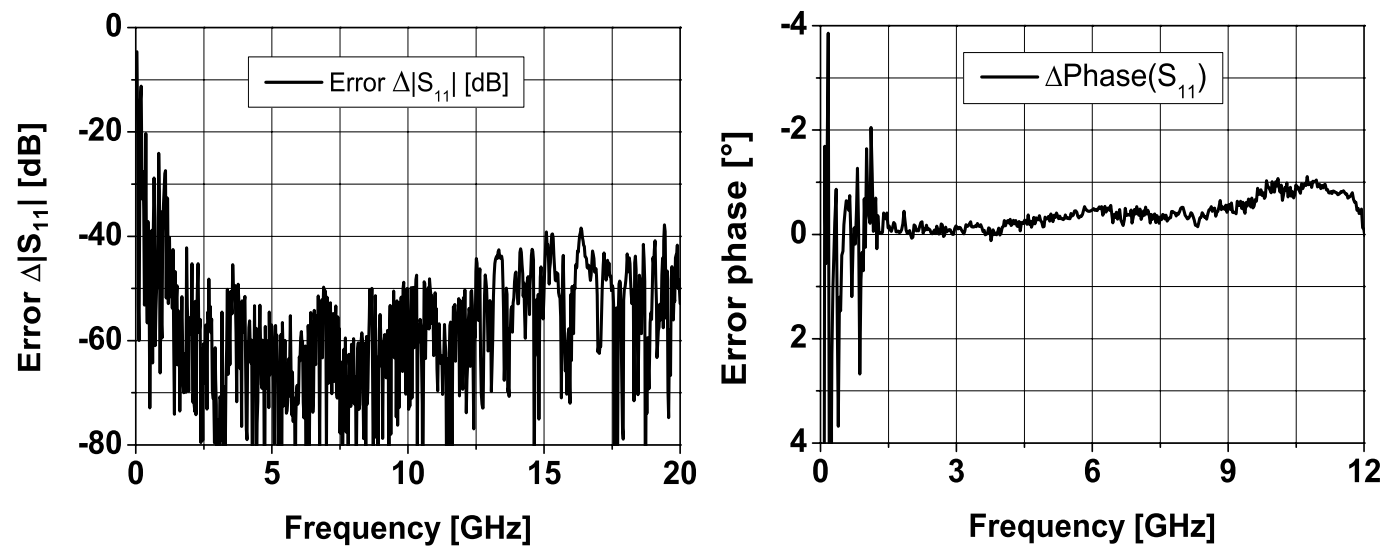

Fig. 13. Comparison of the Parameter $S_{11}$ between conventional and the contactless measurement method, DUT: Filter.
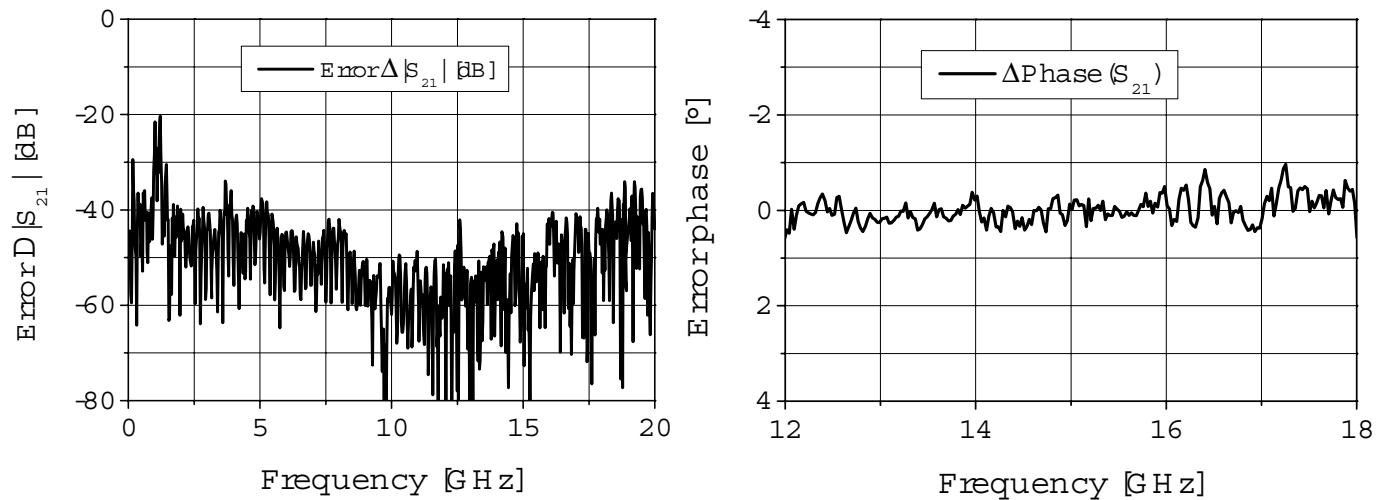

Fig. 14. Comparison of the Parameter $S_{21}$ between the contacted and the contactless measurement method, DUT: Filter.

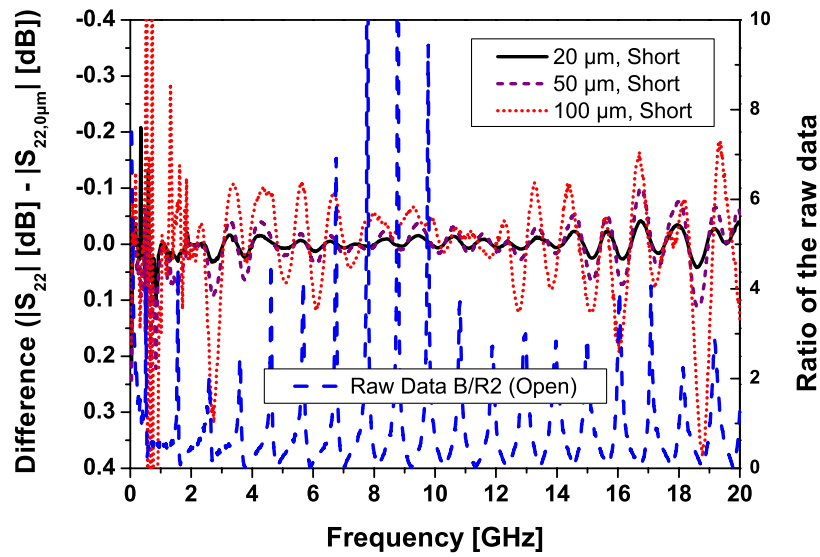

Fig. 15. S-parameters after changing the distance between the probes and the microstrip line after the calibration, DUT: Short.

bration because of the high reflective standards. The quantity of the zero points corresponds to the number of maxima in the curves of the raw data. For using an open standard as termination, the raw data are also plotted in Fig. 15. The raw data is equal to the ratio of the extracted, uncalibrated voltage values at the two measurement positions. Maximum values of the raw data occur at certain frequency positions. This takes place, when the associated probe is located over a voltage minimum on the transmission line. The correlation between the number of zero points and the number of ripple is obvious.

\section{Conclusions}

A contactless measuring system for the determination of S-parameters of two-ports embedded within a complex planar circuit is presented. For this purpose, the internal couplers of a VNA are replaced by four capacitive probes. To determine the S-parameters, conventional calibration methods are used. The contactless system has the advantage that it works wearless. The probe dimension is small with respect to conventional coplanar probes. Also, fast testing becomes possible, due to the not required contacting of the DUT with the probing system. By means of a developed basic model 
the essential system characteristics are analysed. The greatest system dynamics and measurement exactness is achieved by using a probe distance of $\lambda / 4$. Coupling distances in the range of integer multiples of $\lambda / 2$ clearly yield a decrease in system dynamics. Another drawback is the small capacitive coupling in the lower frequency range. An exact measurement in this range is not possible. Furthermore, it is important to keep the probe position constant between calibration and measurement. If the probe position is changed after or during the calibration, the influences of the contactless probes on the transmission line change. This variation causes errors like a decrease of impedance matching or ripples in the S-parameters. In comparison to the conventional determination of the $\mathrm{S}$-parameters using the internal couplers of the VNA, the measurement results of the contactless measuring system showed already very good agreement between $1.2 \mathrm{GHz}$ and $20 \mathrm{GHz}$ with a phase error smaller than $1^{\circ}$ and amplitude deviations small than $-30 \mathrm{~dB}$. Overall, the introduced contactless set-up is an auspicious measuring system for the testing of complex planar circuits. With additional probes in the measurement setup or with the use of a contactless broadband coupler further improvements should be possible.

\section{References}

Bridges, G. E.: Non-contact probing of integrated circuits and packages, IEEE MTT-S International Microwave Symposium Digest, 3, 1805-1808, 2004.

David, G., Schroeder, W., Jager, D., and Wolff, I.: 2D electro-optic probing combined with field theory based multimode wave amplitude extraction: a new approach to on-wafer measurement, IEEE MTT-S International Microwave Symposium Digest, 3, 1049-1052, 1995.

Dudley, R. A., Roddie, A. G., Bannister, D. J., Gifford, A. D., Krems, T., and Facon, P.: Electro-optic S-parameter and electricfield profiling measurement of microwave integrated circuits, IEE Proceedings Science, Measurement and Technology, 146, 3, 117-122, 1999.

Engen, G. F.: Calibration the six-port reflectometer by means of sliding terminations, IEEE Transactions on Microwave Theory and Techniques, MTT-26, 12, 951-957, 1978.
Gao, Y. and Wolff, I.: Measurements of field distributions and scattering parameters in multiconductor structures using an electric field probe, IEEE MTT-S International Microwave Symposium Digest, 3, 1741-1744, 1997.

Gao, Y. and Wolff, I.: Miniature electric near-field probes for measuring 3-D fields in planar microwave circuits, IEEE Transactions on Microwave Theory and Techniques, 46, 7, 907-913, 1998.

Hui, D. and Weikle, R. M.: A non-contacting sampled-line reflectometer for microwave scattering parameter measurements, 64th ARFTG Microwave Measurements Conference, Fall 2004, 131137, 2004.

Mertin, W., Bohm, C., Balk, L. J. and Kubalek, E.: Twodimensional field mapping in MMIC-substrates by electrooptic sampling technique, IEEE MTT-S International Microwave Symposium Digest, 3, 1443-1446, 1992.

Mertin, W., Leyk, A., Behnke, U. and Wittpahl, V.: Contactless gigahertz testing, IEEE Proceedings. International, Test Conference, Paper 33.4, 843-852, 1998.

Osofsky, S. S. and Schwarz, S. E.: Design and performance of a noncontacting probe for measurements on high-frequency planar circuits, IEEE Transactions on Microwave Theory and Techniques, 40, 8, 1701-1708, 1992.

Quardirhi, Z. and Laurin, J. J.: Méthode de measures des paramètres sans contact, CCECE 2003- CCGEI 2003, Montréal, 2003.

Sayil, S., Kerns, D. V. and Kerns, S. E.: A survey contactless measurement and testing technique potentials, IEEE Potentials, 24, 1, 25-28, 2005.

Schiek, B.: Grundlagen der Hochfrequenz-Messtechnik, SpringerVerlag Berlin Heidelberg, ISBN 3-540-64930-1, 1999.

Seitz, H. K., Blacha, A., Clauberg, R., Beha, H. and Feder, J.: Contactless, high-speed waveform measurements on gallium arsenide ICs, IEEE Design \& Test of Computers, 7, 1, 20-25, 1990.

Stenarson, J., Yhland, K., and Wingqvist, C.: An in-circuit noncontacting measurement method for S-parameters and power in planar circuits, IEEE Transactions on Microwave Theory and Techniques, 49, 12, 2567-2572, 2001.

Tabib-Azar, M. and Wang, Y.: Design and fabrication of scanning near-field microwave probes compatible with atomic force microscopy to image embedded nanostructures, IEEE Transactions on Microwave Theory and Techniques, 52, 3, 971-979, 2004.

Zlobin, V. A.: Electron beam technology for power semiconductor device fabrication, Fifth European Conference on Power Electronics and Applications, 2, 73-76, 1993. 